\title{
Explicit Construction of Families of LDPC Codes With No 4-Cycles
}

\author{
Jon-Lark Kim \\ Dept. of Mathematics and Statistics \\ University of Nebraska - Lincoln \\ Lincoln, NE 68588-0323 \\ jlkim@math.unl.edu
}

Uri N. Peled, Irina Perepelitsa, Vera Pless, and Shmuel Friedland

Dept. of Mathematics, Statistics, and Computer Science

University of Illinois at Chicago

Chicago, IL 60607-7045

uripeled@uic.edu

\{irina, pless\}@math.uic.edu

friedlan@uic.edu

May 6, 2003

\begin{abstract}
LDPC codes are serious contenders to Turbo codes in terms of decoding performance. One of the main problems is to give an explicit construction of such codes whose Tanner graphs have known girth. For a prime power $q$ and $m \geq 2$, Lazebnik and Ustimenko construct a $q$-regular bipartite graph $D(m, q)$ on $2 q^{m}$ vertices, which has girth at least $2\lceil m / 2\rceil+4$. We regard these graphs as Tanner graphs of binary codes $\mathrm{LU}(m, q)$. We can determine the dimension and minimum weight of $\mathrm{LU}(2, q)$, and show that the weight of its minimum stopping set is at least $q+2$ for $q$ odd and exactly $q+2$ for $q$ even. We know that $D(2, q)$ has girth 6 and diameter 4 , whereas $D(3, q)$ has girth 8 and diameter 6 . We prove that for an odd prime $p, \operatorname{LU}(3, p)$ is a $\left[p^{3}, k\right]$-code with $k \geq\left(p^{3}-2 p^{2}+3 p-2\right) / 2$. We show that the minimum weight and the weight of the minimum stopping set of $\mathrm{LU}(3, q)$ are at least $2 q$ and they are exactly $2 q$ for many $\mathrm{LU}(3, q)$ codes. We find some interesting LDPC codes by our partial row construction.
\end{abstract}

\section{Introduction}

Low density parity check (LDPC) codes were originally introduced by Gallager [5]. They have again become interesting because of the success of iterative decoding for Turbo codes. LDPC codes are competitors of these codes in performance of iterative decoding algorithms, as their performance approaches the Shannon limit [11]. Tanner's graphical representation of LDPC codes [12] influenced much of the current literature. Most of these codes are constructed randomly, but explicit constructions are needed for implementation purposes as well as for knowing the properties of these codes. We give such constructions based on constructions of graphs with good girth. 
Let $m \geq 2$ be an integer and $q$ a power of a prime. In [9] Lazebnik and Ustimenko construct a family $D(m, q)$ of $q$-regular bipartite graphs on $2 q^{m}$ vertices, with $q^{m}$ vertices called points and $q^{m}$ vertices called lines. Points and lines are elements of $\operatorname{GF}(q)^{m}$ and equations are given in [9], which determine incidence of points and lines. If a point is incident to a line, an edge joins them in $D(m, q)$. It is further shown [9] that when $m$ is odd, $D(m, q)$ has girth at least $m+5$. It also follows from general graph homomorphism results of [10] that the girth of $D(m, q)$ is not less than the girth of $D(m-1, q)$, so for $m$ even, the girth of $D(m, q)$ is at least $m+4$. Thus for all $m$, the girth of $D(m, q)$ is at least $2\lceil m / 2\rceil+4$. We let $H(m, q)$ be the incidence matrix of lines and points of $D(m, q)$, where rows are indexed by lines and columns are indexed by points, and consider $H(m, q)$ and $H(m, q)^{T}$ to be parity check matrices of binary codes of length $q^{m}$ called $L U(m, q)$ codes. In other words, we take $D(m, q)$ to be the Tanner graph [12] of the $\operatorname{LDPC}$ code $\operatorname{LU}(m, q)$ and investigate the properties of these codes. As the rows as well as the columns of $H(m, q)$ are linearly dependent, the dimensions of these codes need to be determined.

The following is shown in [9].

Proposition 1 Any two rows (columns) of $H(m, q)$ have a 1 in at most one common column (row).

This implies that the girth of the graph is at least 6 .

We show that $D(2, q)$ has girth 6 and diameter 4 . We derive the parameters of all $\mathrm{LU}(2, q)$. When $q$ is even we obtain Euclidean geometry codes.

We have computed the dimension of $\mathrm{LU}(3, q)$ codes through $q=25$. We prove that $D(3, q)$ has girth 8 (already shown in [13]). This implies that the minimum weight of $\mathrm{LU}(3, q)$ is at least $2 q[12]$. We show that when $\mathrm{LU}(3, q)$ is derived from $H$, the minimum weight is exactly $2 q$. For $q \geq 3$ the diameter of $D(3, q)$ is 6 [13]. We conjecture the dimension of $\operatorname{LU}(3, q)$ to be $\left(q^{3}-2 q^{2}+3 q-2\right) / 2$ when $q$ is an odd prime power and prove that it is at least $\left(q^{3}-2 q^{2}+3 q-2\right) / 2$ when $q$ is an odd prime. When $q$ is odd we apparently have a family of codes whose rates approach $1 / 2$.

We examined some LU codes for $m=4,5,6$ and 7 and we give our observations. We give a lower bound on the minimum weight of $\operatorname{LU}(m, q)$ in terms of $q$ and $m$ for odd $m$ using Tanner's bound [12].

A stopping set in an LDPC code is a binary row vector having the length of the code that does not have exactly one 1 in common with any row of the parity-check matrix. A minimum stopping set is a nonzero stopping set with minimum weight. Note that any codeword is a stopping set, and therefore the minimum weight of a code is at least the weight of the minimum stopping set. The weight of the minimum stopping set is an important measure of the performance of a code with iterative decoding over the binary erasure channel [4]. We show that for $\operatorname{LU}(2, q)$ the weight of the minimum stopping set is at least $q+2$. It follows from [12] that the weight of the minimum stopping set of $\mathrm{LU}(3, q)$ is at least $2 q$. We show that equality is achieved for $\mathrm{LU}(3, q)$ obtained from $H(3, q)$.

We use a new technique, the partial row construction, to obtain codes with larger rate than $\mathrm{LU}(m, q)$ codes but not smaller girth. We give lists of interesting codes found in this way.

A preliminary version of this paper appeared in [6]. 


\section{$2 \mathrm{LU}(2, \mathrm{q})$ Codes}

Definition 1 ([9]) In $D(2, q)$ a point $(a, b)$ is on a line $[x, y]$ if and only if $y=a x+b$, where $a, b, x, y$ are in $G F(q)$.

We label the rows and columns of $H(2, q)$ with the pairs $[x, y]$ and $(a, b)$ ordered lexicographically under a fixed ordering of $\operatorname{GF}(q)$. If $q$ is a prime, this is the usual ordering; if $q$ is a prime power, we order the elements of $\mathrm{GF}(q)$ in some way, say as powers of a primitive element, with the element 0 first. It can be seen that $H(2, q)$ consists of $q^{2}$ $q \times q$ permutation matrices, where each permutation matrix corresponds to a fixed $a$ and a fixed $x$. If $q$ is a prime, these permutation matrices are circulants. So the first $q$ rows of $H(2, q)$ consist of $q$ permutation matrices, similarly for the next $q$ rows, etc.

We call a row block the set of all rows with fixed $x$, and a column block the set of all columns with fixed $a$.

Proposition 2 No two rows in a row block have a 1 in common, i.e., in the same column. Any two rows from different row blocks have exactly one 1 in common. Similarly for columns.

Proof. This follows from Definition 1.

\section{Example.}

$$
H(2,3)=\left\{H_{i j}\right\}=\left(\begin{array}{ccc|ccc|ccc}
1 & 0 & 0 & 1 & 0 & 0 & 1 & 0 & 0 \\
0 & 1 & 0 & 0 & 1 & 0 & 0 & 1 & 0 \\
0 & 0 & 1 & 0 & 0 & 1 & 0 & 0 & 1 \\
\hline 1 & 0 & 0 & 0 & 0 & 1 & 0 & 1 & 0 \\
0 & 1 & 0 & 1 & 0 & 0 & 0 & 0 & 1 \\
0 & 0 & 1 & 0 & 1 & 0 & 1 & 0 & 0 \\
\hline 1 & 0 & 0 & 0 & 1 & 0 & 0 & 0 & 1 \\
0 & 1 & 0 & 0 & 0 & 1 & 1 & 0 & 0 \\
0 & 0 & 1 & 1 & 0 & 0 & 0 & 1 & 0
\end{array}\right),
$$

where $i, j$ run over the index set $\{00,01,02,10,11,12,20,21,22\}$.

We also note that the code whose parity check matrix is $H(2,3)^{T}$ is the same as the one with parity check matrix $H(2,3)$.

Theorem 1 For $q>2$, all $D(2, q)$ have girth 6 . Also all $D(2, q)$ have diameter 4 .

Proof. By Proposition 1, the girth of $D(2, q)$ is at least 6 . We show that we can find a cycle of length 6 . The first row $r_{1}$ of $H(2, q)$ has a common 1 with the first row $r_{2}$ in the second row block in a column $c_{1}$. There is another column $c_{2}$ with a 1 in $r_{2}$. Column $c_{2}$ has a 1 in a unique row $r_{3}$ of the third row block. Row $r_{3}$ must have a common 1 with row $r_{1}$, but not in $c_{1}$ (or else $r_{2}$ and $r_{3}$ would have two common 1 's) and not in $c_{2}$ (or else $r_{1}$ and $r_{2}$ would have two common 1's). So there is a third column $c_{3}$ having common 1 's with $r_{1}$ and $r_{2}$. Then $r_{1}-c_{1}-r_{2}-c_{2}-r_{3}-c_{3}-r_{1}$ is a cycle of length 6 in $D(2, q)$.

Two rows in different row blocks and two columns in different column blocks have distance 2 from each other. A row and a column have distance 1 or 3 , and two rows or columns in the same row or column block have distance 4. Hence the diameter of $D(2, q)$ is 4 . 
Theorem 2 If $q$ is odd, the two $L U(2, q)$ codes are the same $\left[q^{2}, q-1,2 q\right]$ code, whose group has order $(q !)^{q+1}$.

Proof. We construct a canonical spanning set of $\mathrm{LU}(2, q)^{\perp}$. If we add all the rows in any row block of $H(2, q)$, we obtain the all-one vector. If we add up all the rows in $H(2, q)$ that have 1 in a fixed column, we will be adding one row from each row block. If for example the column is the first column, the resulting sum will be

$$
\underbrace{10 \ldots 0}_{q} \underbrace{11 \ldots 1}_{q} \cdots \underbrace{11 \ldots 1}_{q} \text {. }
$$

This is so since no two rows in a row block have a 1 in common by Proposition 2 and since $q$ is odd. Hence $\mathrm{LU}(2, q)^{\perp}$ contains all the rows of the following matrix

$$
A=\left(\begin{array}{ccccc}
E & 0 & \ldots & \ldots & 0 \\
0 & E & 0 & \ldots & 0 \\
\vdots & \vdots & \vdots & \vdots & \vdots \\
0 & 0 & 0 & \ldots & E \\
1 & 1 & 1 & \ldots & 1
\end{array}\right)
$$

where $E=I+J$ with $I$ the $q \times q$ identity matrix and $J$ the $q \times q$ all-one matrix, and where 1 is the all-one row vector of length $q$.

As $E$ has rank $q-1, A$ generates a code of dimension $q(q-1)+1$ (the all-one vector of odd weight is not equal to any sum of previous rows, as all such sums have even weight). It is not hard to see that the rows of $A$ span $\mathrm{LU}(2, q)^{\perp}$, as we can express any row of $H(2, q)$ as a sum of these rows. Hence for $q$ odd, $\operatorname{dim}(\mathrm{LU}(2, q))=q^{2}-(q(q-1)+1)=q-1$.

From the generating set $A$ of $\operatorname{LU}(2, q)^{\perp}$, we see that the group of this code consists of $\operatorname{Sym}(q)$ operating independently on each column block of $q$ elements, and another $\operatorname{Sym}(q)$ permuting the $q$ column blocks. Hence for $q$ odd, the group of $\operatorname{LU}(2, q)$ has order $(q !)^{q+1}$.

We can also determine the minimum weight of $\mathrm{LU}(2, q)$ by looking at $A$. The dual of each $E$ is the all-one vector of length $q$. But as the all-one vector of length $q^{2}$ is in $\mathrm{LU}(2, q)^{\perp}$, every vector in $\mathrm{LU}(2, q)$ has even weight. So the minimum weight of $\operatorname{LU}(2, q)$ is $2 q$. $\mathrm{LU}(2, q)$ can be regarded as all even-weight row vectors made out of all-0 and all-1 blocks of length $q$. As we also get $A$ as above for $H(2, q)^{T}$, the two $\operatorname{LU}(2, q)$ codes are in fact the same.

When $q$ is even, we get interesting results.

Lemma $1 H\left(2,2^{s}\right)$ is the incidence matrix of $2^{2 s}$ points and $2^{2 s}$ lines consisting of parallel classes from the affine plane $A G\left(2,2^{s}\right)$. Further, the code $C$ generated by $H\left(2,2^{s}\right)$ contains all the lines of this affine plane. The same results hold for $H\left(2,2^{s}\right)^{T}$.

Proof. Each row of $H\left(2,2^{s}\right)$ has weight $2^{s}$, the weight of a line in an affine geometry from a projective plane $\mathrm{PG}\left(2,2^{s}\right)$ of order $2^{s}$. We regard these rows as lines of the geometry. By Proposition 2, each row block is a parallel class of lines. There are $2^{s}$ such blocks in $H\left(2,2^{s}\right)$. The affine plane has $2^{s}+1$ parallel classes of lines. This last parallel class consists of the $2^{s}$ row vectors each of which is the all-one vector in a fixed column block and zero outside the block. We show as follows that these vectors are in $C$. If we add up all the rows of $H\left(2,2^{s}\right)$ that have a 1 in their first position, we get $\underbrace{0 \ldots 0}_{2^{s}} \underbrace{1 \ldots 1}_{2^{s}} \cdots \underbrace{1 \ldots 1}_{2^{s}}$ by Proposition 2 and since $2^{s}$ is even. Adding the all-one vector, which is the sum of 
the rows in any row block, we get $\underbrace{1 \ldots 1}_{2^{s}} \underbrace{0 \ldots 0}_{2^{s}} \ldots \underbrace{0 \ldots 0}_{2^{s}}$, a line in the missing parallel class. We can get the rest of the lines similarly. The fact that this affine plane comes from $\mathrm{PG}\left(2,2^{s}\right)$ follows from the equations in Definition 1. The same proof works for $H\left(2,2^{s}\right)^{T}$.

Theorem $3 \mathrm{LU}\left(2,2^{s}\right)$ are $\left[2^{2 s}, 2^{2 s}-3^{s}, 2^{s}+2\right]$ codes.

Proof. We only consider $H\left(2,2^{s}\right)$. It is known [1] that the incidence matrix of an affine plane of order $2^{s}$ generates a $\left[2^{2 s}, 3^{s}\right]$ binary code $C$. By Lemma $1 \mathrm{LU}\left(2,2^{s}\right)$ is the code $C$. The minimum-weight vectors of $C^{\perp}$ contain all the ovals of the corresponding projective plane [8], and as there exist ovals disjoint from the line at $\infty$, the minimum weight of $\mathrm{LU}\left(2,2^{s}\right)$ is $2^{s}+2$. Hence $\operatorname{LU}\left(2,2^{s}\right)$ is a $\left[2^{2 s}, 2^{2 s}-3^{s}, 2^{s}+2\right]$ code.

Theorem 4 The weight of the minimum stopping set of $L U(2, q)$ is at least $q+2$.

Proof. We denote by $r_{i}$ and $c_{i}$ the $i$-th row and the $i$-th column of $H(2, q)$, respectively. Let $v$ be a minimum stopping set of $\mathrm{LU}(2, q)$. Pick some component of $v$ that is equal to 1 , say $v_{1}=1$. The column $c_{1}$ has $q 1$ 's, say in rows $r_{1}, \ldots, r_{q}$. Since $r_{1}, \ldots, r_{q}$ have a common 1 with $v$ in $c_{1}$, each of them has another common 1 with $v$, and no two of them can have the other common 1 with $v$ in the same column by Proposition 1 . Therefore we may assume that $r_{i}$ has a common 1 with $v$ in $c_{i+1}$ for $i=1, \ldots, q$. For each $i=2, \ldots, q+1, c_{i}$ has a common 1 with $c_{1}$ in $r_{i-1}$, and therefore by Proposition 2 the $c_{i}$ belong to different column blocks than the column block containing $c_{1}$. There are only $q$ column blocks, and therefore two of $c_{1}, \ldots, c_{q+1}$ are in the same column block. By the above both of them are distinct from $c_{1}$, so we may assume that $c_{q}$ and $c_{q+1}$ are in the same column block. By Proposition $2 c_{q+1}$ does not have a common 1 with $c_{q}$. Column $c_{q+1}$ must have 0's in $r_{1}, \ldots, r_{q-1}$, otherwise it would have two common 1's with $c_{1}$. Since it has weight $q$, we may assume that it has 1 's in $r_{q+1}, \ldots, r_{2 q-1}$. Each of the $q-1$ rows $r_{q+1}, \ldots, r_{2 q-1}$ has a common 1 with $v$ in $c_{q+1}$, and therefore must have another common 1 with it, but not in $c_{1}$ (since $c_{1}$ already has its $q$ 1's in $r_{1}, \ldots, r_{q}$ ), and not in $c_{q}$ (since $c_{q+1}$ and $c_{q}$ do not have common 1's). Furthermore, no two of these $q-1$ rows can have a common 1 with $v$ in the same column (since both of them already have a common 1 with it in $c_{q+1}$ ). Therefore at most $q-2$ of them can have a common 1 with $v$ in $c_{2}, \ldots, c_{q-1}$, and one of them must have a common 1 with $v$ outside $c_{1}, \ldots, c_{q+1}$, say in $c_{q+2}$. Thus $q+2$ components of $v$ are 1 .

It follows from Theorems 3 and 4 that for $q$ even, the weight of the minimum stopping set of $\mathrm{LU}(2, q)$ is $q+2$.

In [7] families of LDPC codes with girth 6 were constructed from finite geometries. One of these families of Euclidean geometry codes has parameters $\left[2^{2 s}-1,2^{2 s}-3^{s}, 2^{s}+1\right]$. We extended two of these codes for $s=2$ and $s=3$ and (using Magma [3]) found that they are equivalent to $\mathrm{LU}(2,4)$ and $\mathrm{LU}(2,8)$. This will be so in general since both families of codes are constructed from $\mathrm{PG}\left(2,2^{s}\right)$. However, the two families could have different decoding performance as the parity check matrices used are different. In fact, the parity check matrices in [7] are cyclic, whereas ours are not. 


\section{$3 \mathrm{LU}(3, \mathrm{q})$ Codes}

Definition 2 ([9]) In $D(3, q)$, a point $(a, b, c)$ is incident with a line $[x, y, z]$ if and only if $y=a x+b$ and $z=a y+c$, where $a, b, c, x, y, z$ are in $G F(q)$.

We investigated the parameters of the $\mathrm{LU}(3, q)$ codes for $q=2$ up to $q=25$ by Magma. By [9], all the Tanner graphs of the $\mathrm{LU}(3, q)$ codes have girth at least $3+5=8$. We give a simple proof that the girth is exactly 8. By [13], $D(3, q)$ has diameter 6 for $q \geq 3$. $D(3,2)$ is disconnected; it is a union of two 8-cycles. So $\operatorname{LU}(3,2)$ is the direct sum of two $[4,1,4]$ codes, each of which is an $\mathrm{LU}(2,2)$ code.

Theorem 5 ([13]) $D(3, q)$ has girth 8. Its diameter is 6 if $q>2$.

Proof. Since by [9] we know that the girth of $D(3, q)$ is at least 8 , finding one 8-cycle shows that the girth is 8 . It is not hard to check that $(000)-[000]-(100)-[111]-$ $(011)-[011]-(110)-[-100]-(000)$ is an 8-cycle in $D(3, q) . D(3, q)$ has diameter 6 for $q>2$ [13, Theorem 3.9].

Theorem 6 For $p$ an odd prime, $L U(3, p)$ is a $\left[p^{3}, k\right]$-code with $k \geq\left(p^{3}-2 p^{2}+3 p-2\right) / 2$. Proof. See Appendix.

Conjecture For odd $q, L U(3, q)$ is a $\left[q^{3},\left(q^{3}-2 q^{2}+3 q-2\right) / 2\right]$ code.

We verified this for all of the $\mathrm{LU}(3, q)$ codes for all odd $q$ from 3 until 25 . If this is true, then the rate of these codes approach $1 / 2$ as the odd $q$ gets large. We noticed that for $q=3$ and $q=5$, the two $\operatorname{LU}(3, q)$ codes we obtain from $H(3, q)$ and its transpose have different minimum weights. We checked by Magma that for $q=4$ the two codes are equivalent. See Table 1. For $q \geq 7$, we were unable to determine the minimum weight of $\mathrm{LU}(3, q)$ derived from $H^{T}$.

Table 1: Parameters of $\mathrm{LU}(3, q)$ codes for $q=3,4,5$.

\begin{tabular}{l|ccc}
$q$ & 3 & 4 & 5 \\
\hline$H$ & {$[27,8,6]$} & {$[64,22,8]$} & {$[125,44,10]$} \\
$H^{T}$ & {$[27,8,8]$} & {$[64,22,8]$} & {$[125,44,20]$}
\end{tabular}

Theorem 7 The minimum weight and the weight of the minimum stopping set of $L U(3, q)$ are at least $2 q$.

Proof. The bound on the minimum weight follows from [12, Theorem 2] since we know that the girth of $\mathrm{LU}(3, q)$ is 8 . However, Tanner's proof in [12] also holds for the minimum stopping set.

Theorem 8 The minimum weight of $L U(3, q)$ obtained from $H$ is $2 q$. Consequently the weight of the minimum stopping set of $L U(3, q)$ obtained from $H$ is also $2 q$. 
Proof. By Theorem 7 the minimum weight of $\mathrm{LU}(3, q)$ is at least $2 q$. Therefore finding a codeword of weight $2 q$ in $\mathrm{LU}(3, q)$ obtained from $H$ will complete the proof of the theorem.

Let $\alpha$ be a primitive element of $G F(q)$. Consider a word $W$ containing the following points:

1. $\left(\alpha^{-2}, 0,0\right)$, which lies only on lines of the form $\left[x, \alpha^{-2} x, \alpha^{-4} x\right]$;

2. $\left(\alpha^{-2}, \alpha^{k}, \alpha^{k-1}\right), 0 \leq k \leq q-2$, which lies only on lines of the form $\left[x, \alpha^{-2} x+\right.$ $\left.\alpha^{k}, \alpha^{-4} x+\alpha^{k-2}+\alpha^{k-1}\right]$

3. $\left(\alpha^{-1}, 0,0\right)$, which lies only on lines of the form $\left[x, \alpha^{-1} x, \alpha^{-2} x\right]$;

4. $\left(\alpha^{-1}, \alpha^{l+2}, \alpha^{l}\right), 0 \leq l \leq q-2$, which lies only on lines of the form $\left[x, \alpha^{-1} x+\right.$ $\left.\alpha^{l+2}, \alpha^{-2} x+\alpha^{l+1}+\alpha^{l}\right]$.

These $2 q$ points are distinct, so $W$ has weight $2 q$. We show that $W$ is a codeword.

Lines of the form 1 and 2 never coincide. Lines of the form 3 and 4 never coincide. A line of the form 1 coincides with a line of the form 3 if and only if $x=0$; it coincides with a line of the form 4 if and only if $x=\frac{\alpha^{l+4}}{1-\alpha}$. A line of the form 2 coincides with a line of the form 3 if and only if $x=\frac{\alpha^{k+2}}{1-\alpha}$; it coincides with a line of the form 4 if and only if $x=\frac{\alpha^{l+4}-\alpha^{k+2}}{1-\alpha}$. Now let $L$ be a line of the form 1 . If $x=0$, then $L$ is also of the form 3 , but not of the forms 2 or 4 . So $L$ contains only two points of $W$, namely $\left(\alpha^{-2}, 0,0\right)$ and $\left(\alpha^{-1}, 0,0\right)$. If $x \neq 0$, then $L$ coincides with a unique line of the form 4 given by the unique $l$ such that $x=\frac{\alpha^{l+4}}{1-\alpha}$, but not with any line of the form 2 or 3 . So $L$ contains only two points of $W$, namely $\left(\alpha^{-2}, 0,0\right)$ and $\left(\alpha^{-1}, \alpha^{l+2}, \alpha^{l}\right)$. Let $L$ be a line of the form 2 . Then $L$ does not coincide with any line of the form 1 . If $x \neq 0$, then $L$ coincides with a unique line of the form 3 given by the unique $k$ such that $x=\frac{\alpha^{k+2}}{1-\alpha}$; in that case $L$ cannot coincide with a line of the form 4 , since lines of the forms 3 and 4 never coincide. So $L$ contains only two points of $W$, namely $\left(\alpha^{-2}, \alpha^{k}, \alpha^{k-1}\right)$ and $\left(\alpha^{-1}, 0,0\right)$. If $x=0$, then $L$ cannot coincide with a line of the form 3 , but it does coincide with a unique line of the form 4 given by $l=k-2$, so again $L$ contains only two points of $W$. Let $L$ be a line of the form 3. If $x=0$, then $L$ coincides with a unique line of type 1 and no line of type 2 or 4 . If $x \neq 0$, then $L$ coincides with a unique line of type 2 and no line of type 1 or 4. In any case $L$ contains only two points of $W$. Let $L$ be a line of type 4 . If $x \neq 0$, then $L$ coincides with a unique line of type 1 and no line of type 2 or 3 . If $x=0$, then $L$ coincides with a unique line of type 2 and no line of type 1 or 3 . In any case $L$ contains only two points of $W$. We have shown that each line containing a point of $W$ contains precisely two points of $W$. Therefore $W$ is a codeword.

From the examples above for $q=3$ and $q=5$ it seems that for odd $q, \mathrm{LU}(3, q)$ derived from $H^{T}$ has minimum weight larger than $2 q$.

\section{The Partial Row Construction}

In investigating the LU codes, we found many that have low rates. We decided to consider those codes whose parity check matrices consist of the first $i$ rows of $H(m, q)$, where $i<q^{m}$ (we order the rows and columns lexicographically as in Section 2). We call this the partial row construction. If we consider a code $C$ whose parity check matrix 
consists of the first $i$ rows of $H(m, q)$, then the rate of $C$ may stay the same or be higher than that of $\mathrm{LU}(m, q)$, the girth of its Tanner graph may stay the same or go up, but the minimum weight might go down. We found a number of interesting LDPC codes by the partial row construction for $m=2$, which we list in Table 2 .

Table 2: LDPC codes obtained by the partial row construction from $\mathrm{LU}(2, q)$ codes.

\begin{tabular}{r|lcc}
$q$ & {$[n, k, d]$} & (girth, diameter) & \# of rows of $H(2, q)$ \\
\hline 3 & {$[9,4,4]$} & $(8,4)$ & 6 \\
4 & {$[16,9,4]$} & $(8,4)$ & 8 \\
5 & {$[25,12,6]$} & $(6,4)$ & $14-15$ \\
7 & {$[49,24,8]$} & $(6,4)$ & $27-28$ \\
8 & {$[64,37,10]$} & $(6,4)$ & $57-64$ \\
9 & {$[81,32,16]$} & $(6,4)$ & $53-54$ \\
11 & {$[121,84,8]$} & $(6,4)$ & 39
\end{tabular}

When $q=8$ and the number of rows is 64 , this code is $\operatorname{LU}(2,8)$. Note that the $[9,4,4]$, $[16,9,4]$ and $[64,37,10]$ codes are optimal, whereas the $[25,12,6]$ and $[81,32,16]$ codes are just 2 short of being optimal [2]. The other two codes have minimum weight 4 less than the optimal codes. The parity check matrix for the $[9,4,4]$ code consists of the first 6 rows of $H(2,3)$ given in the example in Section 2.

We also improve the rate while maintaining the minimum weight, girth and diameter for $\mathrm{LU}(2, q)$ codes by the partial row construction. We list them below.

$$
\begin{array}{llll}
\text { old } & {[25,4,10]} & {[49,6,14]} & {[81,8,18]} \\
\text { new } & {[25,6,10]} & {[49,10,14]} & {[81,14,18]}
\end{array}
$$

We obtain interesting $\mathrm{LDPC}$ codes from $\mathrm{LU}(3, q)$ codes by the partial row construction. They are listed in Table 3. Many have larger girths than the $\mathrm{LU}(3, q)$ code. We list only those where we were able to find the minimum distance.

Table 3: Codes from $\mathrm{LU}(3, q)$ codes by the partial row construction.

\begin{tabular}{c|lccc}
$q$ & {$[n, k, d]$} & (girth, diameter) & $H(3, q)$ or $H^{T}(3, q)$ & \# of rows \\
\hline & & & & \\
3 & {$[27,12,4]$} & $(16,10)$ & $H$ & 15 \\
3 & {$[27,10,6]$} & $(12,8)$ & $H$ & 18 \\
4 & {$[64,35,4]$} & $(8,10)$ & $H^{T}$ & 33 \\
5 & {$[125,54,14]$} & $(8,6)$ & $H^{T}$ & 85 \\
5 & {$[125,47,20]$} & $(8,6)$ & $H^{T}$ & 105
\end{tabular}

\section{The Cases $\mathrm{m}=4,5,6,7$}

The equations for $D(m, q)$ for $m=4,5,6,7$ are considerably more complicated than for $m=2,3$. They can be found in [9].

$D(m, 2)$ is disconnected for $m=3,4,5,6$ and 7 . In [9], the authors state that they and A.J. Woldar proved that for $m \geq 6$, all $D(m, q)$ are disconnected. In fact, in [13, pg. 79] it is shown that for $q=3$ and for $q>4, D(m, q)$ has $q^{t-1}$ connected components, 
where $t=\left\lfloor\frac{m+2}{4}\right\rfloor$, and for $m \geq 4 D(m, 4)$ has $4^{t}$ connected components. So even though the graphs $D(m, q)$ have large girth (at least $2\lceil m / 2\rceil+4)$, the large length of the code and the disconnectedness makes them more difficult to use as Tanner graphs of LDPC codes. We do know the following.

Theorem 9 When $D(m, q)$ is disconnected, it is a union of isomorphic connected subgraphs. In this case $L U(m, q)$ is a direct sum of equivalent codes each of which has its parity check matrix from the incidence matrix of a connected component subgraph.

Proof. When $D(m, q)$ is disconnected, it is a union of isomorphic connected subgraphs since the group of $D(m, q)$ is edge-transitive [9]. This is so because if an automorphism of a graph maps an edge $e$ into an edge $f$, then it maps the connected component of $e$ onto the connected component of $f$. So if for every two edges of $D(m, q)$ there is an automorphism mapping one onto the other, then for every two connected components there is an automorphism mapping one onto the other.

We can reorder the rows and the columns of $H(m, q)$ by putting the rows and the columns of the first connected component first, the rows and the columns of the second connected component second, etc. From this we can see that $\mathrm{LU}(m, q)$ is a direct sum of codes. Codes corresponding to distinct connected components are equivalent, since the connected components are isomorphic.

We found directly that $\mathrm{LU}(4,4)$, a $[256,88,8]$ code, is a direct sum of four $[64,22,8]$ codes of girth 8 and diameter 6; and that LU(5,4), a [1024,216] code, is a direct sum of four $[256,54]$ codes of girth 10 and diameter 8 .

Since we have a lower bound of $2\lceil m / 2\rceil+4$ on the girth, a lower bound on the minimum distance can be obtained.

Theorem 10 The minimum distance $d$ of $L U(m, q)$ satisfies

$$
d \geq \begin{cases}2 \frac{(q-1)^{\lfloor m / 4\rfloor+1}-1}{q-2}, & m \equiv 0 \bmod 4 \\ 2 \frac{(q-1)^{\lfloor m / 4\rfloor+2}-1}{q-2}, & m \equiv 3 \bmod 4 \\ 2 \frac{(q-1)^{\lfloor m / 4\rfloor+1}-1}{q-2}+\frac{2}{q}(q-1)^{\lfloor m / 4\rfloor+1}, & m \equiv 1,2 \bmod 4 .\end{cases}
$$

When $q=2$, the fraction $\frac{(q-1)^{\lfloor m / 4\rfloor+1}-1}{q-2}$ is understood to be $\lfloor m / 4\rfloor+1$, and $\frac{(q-1)^{\lfloor m / 4\rfloor+2}-1}{q-2}$ to be $\lfloor m / 4\rfloor+2$. The same bound holds for the weight of the minimum stopping set.

Proof. This bound on the minimum weight follows from the proof of [12, Theorem 2], using the fact that the column sums of $H(m, q)$ are $q$. However, Tanner's proof in [12] also holds for the minimum stopping set.

In particular, $d \geq 2 q$ for $m=3,4 ; d \geq 4 q-3$ for $m=5,6 ; d \geq 2\left(q^{2}-q+1\right)$ for $m=7,8$; and $d \geq 4(q-1)^{2}+4$ for $m=9,10$ and $q>2$.

\section{Appendix: Proof of Theorem 6}

We begin by a series of lemmas determining the ranks of certain matrices. Then we proceed to obtain an upper bound on the rank of $H(3, p)$ when $p$ is an odd prime, using the previous results. 
Lemma 2 Let $x_{1}, \ldots, x_{m}$ be indeterminates, and consider the $m \times m$ matrix

$$
A\left(x_{1}, \ldots, x_{m}\right)=\left(a_{i j}\right)_{1}^{m}, \quad a_{i j}=\left(x_{j}\right)^{i}-1
$$

over $\mathbb{Z}$. Then

$$
\operatorname{det} A\left(x_{1}, \ldots, x_{m}\right)=\prod_{0 \leq i<j \leq m}\left(x_{j}-x_{i}\right), \quad \text { where } x_{0}=1 .
$$

Proof. Consider the $(m+1) \times(m+1)$ Vandermonde matrix with variables $x_{0}, x_{1}, \ldots, x_{m}$, and substitute $x_{0}=1$. The determinant of the resulting matrix is given by the righthand side of (1). If we subtract the first row from all other rows and then expand the determinant by the first column, we see that it is also equal to the left-hand side of (1).

Lemma 3 If $t$ is an indeterminate, then

$$
\operatorname{det}\left(t^{i \cdot j}-1\right)_{1}^{m}=\prod_{0 \leq i<j \leq m}\left(t^{j}-t^{i}\right) .
$$

Proof. This is a special case of Lemma 2, where $x_{j}=t^{j}$ for all $j=1, \ldots, m$.

Lemma 4 Let $\zeta \in \mathbb{C}$ be a primitive $s$-th root of unity, and consider the matrix $A(m, \zeta)=$ $\left(\zeta^{i j}-1\right)_{1}^{m}$. Then the rank of $A(m, \zeta)$ is $\min (s-1, m)$.

Proof. This is trivial for $s=1$, where $\zeta=1$ and $A(m, \zeta)=0$, so we assume $s>1$. Our first case is $m \leq s-1$. Then $\zeta^{0}, \ldots, \zeta^{m}$ are all distinct, and so Lemma 3 shows that the rank of $A(m, \zeta)$ is $m$. Our second case is $m \geq s$. Then row $i$ of $A(m, \zeta)$ vanishes for each $1 \leq i \leq m$ that is divisible by $s$. If $1 \leq i \leq m$ is not divisible by $s$, we write $i=s q+r$ with $1 \leq r<s$, and then row $i$ of $A(m, \zeta)$ is equal to row $r$. It follows that the row-space of $A(m, \zeta)$ is spanned by the first $s-1$ rows, which are linearly independent by the first case. Hence the rank of $A(m, \zeta)$ is $s-1$.

For a positive integer $q$, we denote by $I_{k}$ an identity matrix of order $q$ whose rows are cyclically shifted $k$ positions to the right, i.e., $\left(I_{k}\right)_{i, j}=1$ if $j-i \equiv k \bmod q, 0$ otherwise. For example, with $q=5$, we have

$$
I_{2}=\left(\begin{array}{ccccc}
0 & 0 & 1 & 0 & 0 \\
0 & 0 & 0 & 1 & 0 \\
0 & 0 & 0 & 0 & 1 \\
1 & 0 & 0 & 0 & 0 \\
0 & 1 & 0 & 0 & 0
\end{array}\right)
$$

Note that $I_{k}=I_{1}^{k}$ for all integers $k$ (not necessarily positive, so for example, $I_{2}$ above is equal to $I_{-3}$ ). For positive integers $m$ and $q$, we denote by $M$ the block matrix with $m$ block rows and $m$ block columns, where the $(i, j)$ block is $I_{i \cdot j}-I_{0}$ of order $q$. For example, with $m=3$, we have

$$
M=\left(\begin{array}{ccc}
I_{1}-I_{0} & I_{2}-I_{0} & I_{3}-I_{0} \\
I_{2}-I_{0} & I_{4}-I_{0} & I_{6}-I_{0} \\
I_{3}-I_{0} & I_{6}-I_{0} & I_{9}-I_{0}
\end{array}\right)
$$


where the subscripts in the $I_{k}$ can be reduced $\bmod q$ if desired. Recall that the Euler function $\phi(n)$ is defined by

$$
\phi(n)=|\{k \in \mathbb{Z} \mid 1 \leq k \leq n, \operatorname{gcd}(k, n)=1\}| .
$$

Lemma 5 With $M$ defined above, the rank of $M$ over $\mathbb{C}$ is

$$
\sum_{\substack{1 \leq s \leq q \\ s \mid q}} \phi(s) \min (s-1, m) .
$$

Proof. If $\zeta$ is a $q$-th root of unity, then $\left(1, \zeta, \ldots, \zeta^{q-1}\right)^{T}$ is an eigenvector of $I_{1}$ with eigenvalue $\zeta$. Therefore if $X=\left(\zeta_{i}^{j-1}\right)_{i, j=1}^{q}$ is the Vandermonde matrix corresponding to all the $q$-th roots of unity $\zeta_{1}, \ldots, \zeta_{q}$, then

$$
I_{1}=X D X^{-1}, \quad \text { where } D=\operatorname{diag}\left(\zeta_{1}, \ldots, \zeta_{q}\right) \text {. }
$$

Let $X_{m}$ be the $m \times m$ block diagonal matrix $\operatorname{diag}(X, \ldots, X)$. The matrix $X_{m}^{-1} M X_{m}$ has the same rank as $M$. By (4), the $(i, j)$ block of $X_{m}^{-1} M X_{m}$ is the $q \times q$ diagonal matrix $\operatorname{diag}\left(\zeta_{1}^{i j}-1, \ldots, \zeta_{q}^{i j}-1\right)$. By permuting the rows of $X_{m}^{-1} M X_{m}$ so that rows $1, q+1, \ldots,(m-1) q+1$ come first, then rows $2, q+2, \ldots(m-1) q+2$, and so on, and likewise for columns, we see that $X_{m}^{-1} M X_{m}$ is permutationally similar to the block diagonal matrix

$$
\bigoplus_{k=1}^{q}\left(\zeta_{k}^{i j}-1\right)_{i, j=1}^{m}
$$

( $m \times m$ blocks, $q$ block rows and $q$ block columns). Thus the rank of $M$ is the sum of the ranks of the diagonal blocks in (5). To find the rank of the $k$-th diagonal block, let $\zeta_{k}$ be a primitive $s$-th root of unity, so that $s \mid q$. By Lemma 4 , the rank of the $k$-th diagonal block is $\min (s-1, m)$. Since there are exactly $\phi(s)$ primitive $s$-th roots of unity, the sum of the ranks of the diagonal blocks is given by (3).

We now consider $M$ as a binary matrix, so that its $(i, j)$ block can be written as $I_{i j}+I_{0}$.

Lemma 6 If $q$ is an odd positive integer, then the binary rank of $M$ defined above is again given by (3).

Proof. Consider the polynomial $f(t)=t^{q}-1$ over GF(2). Since $q$ is odd, we have $f^{\prime}(t)=q t^{q-1}=t^{q-1}$, so $f$ and $f^{\prime}$ are relatively prime in $\operatorname{GF}(2)[t]$, and so the roots of $f$ are simple. Let $\mathbb{F}$ be a finite extension field of $\mathrm{GF}(2)$ such that $f(t)$ splits over $\mathbb{F}$ : $f(t)=\left(t-\zeta_{1}\right) \cdots\left(t-\zeta_{q}\right)$. Thus $\zeta_{1}, \ldots, \zeta_{q}$ are $q$-th roots of unity in $\mathbb{F}$. They are distinct since $f$ has simple roots, so they comprise all the $q$-th roots of unity in $\mathbb{F}$. We can then repeat the entire argument from Lemma 2 through Lemma 5 in $\mathbb{F}$ instead of in $\mathbb{C}$, and we obtain that the rank of $M$ over $\mathbb{F}$ is given by (3). However, the rank over $\mathbb{F}$ is the same as the binary rank, since the entries of $M$ are in $\mathrm{GF}(2)$, and the rank is the largest order of a nonzero minor.

Lemma 7 If $p$ is an odd prime and $1 \leq m \leq p-1$, then the binary rank of $M$ defined above is $m(p-1)$.

Proof. This is a special case of Lemma 6. 
We now shift our attention to $H(3, q)^{T}$. Its rows are indexed by triples $(a, b, c)$ with $a, b, c \in \mathrm{GF}(q)$, and we let $R(a, b, c)$ denote the row indexed by $(a, b, c)$. Similarly, the columns are indexed by triples $[x, y, z]$ with $x, y, z \in \mathrm{GF}(q)$, and we let $C[x, y, z]$ denote the corresponding column. As before, we call a row block the set of all $q^{2}$ rows $R(a, b, c)$ with the same $a$, and we also call a row subblock a set of all $q$ rows $R(a, b, c)$ with the same $(a, b)$. Similarly for column blocks and column subblocks.

Recall from Definition 2 that $R(a, b, c)$ meets $C[x, y, z]$ (i.e., $H(3, q)^{T}$ has a 1 in this row and column) if and only if $y=a x+b$ and $z=a y+c$. It follows that the $q \times q$ intersection of a row subblock and a column subblock is either zero or a permutation matrix. In the latter case, we say that the row subblock and the column subblock meet. Moreover, each row subblock meets exactly one column subblock of each column block.

Lemma 8 For each $(a, b, c)$ and each $a^{\prime} \neq a$, one has

$$
\sum_{b^{\prime}} R\left(a^{\prime}, b^{\prime}, a b^{\prime}-a^{\prime} b+c\right)+\sum_{b^{\prime \prime}} R\left(a, b^{\prime \prime}, a^{\prime}\left(b^{\prime \prime}-b\right)+c\right)=0 .
$$

Proof. We need to show that each column $C[x, y, z]$ meets an even number of rows involved in the left-hand side of (6). Column $C[x, y, z]$ meets $R\left(a^{\prime}, b^{\prime}, a b^{\prime}-a^{\prime} b+c\right)$ if and only if $y=a^{\prime} x+b^{\prime}$ and $z=a^{\prime} y+a b^{\prime}-a^{\prime} b+c$. These two equations can be satisfied by at most one value of $b^{\prime}$, and such $b^{\prime}$ exists if and only if $z=a^{\prime} y+a\left(y-a^{\prime} x\right)-a^{\prime} b+c$. Similarly, $C[x, y, z]$ meets $R\left(a, b^{\prime \prime}, a^{\prime}\left(b^{\prime \prime}-b\right)+c\right)$ if and only if $y=a x+b^{\prime \prime}$ and $z=a y+a^{\prime}\left(b^{\prime \prime}-b\right)+c$. Again, these two equations can be satisfied by at most one value of $b^{\prime \prime}$, and such $b^{\prime \prime}$ exists if and only if $z=a y+a^{\prime}(y-a x-b)+c$. Since the conditions of existence of $b^{\prime}$ and of $b^{\prime \prime}$ are equivalent, $C[x, y, z]$ meets exactly zero or exactly two rows involved in the left-hand side of (6).

From now on we assume that $p$ is an odd prime. Then any nonzero intersection of a row subblock and a column subblock has the form of a circulant $I_{c}$ of order $p$.

Definition $3 A$ vector of the form $\sum_{c} R(a, b, c)$, i.e., the sum of the rows in a given row subblock, is said to be of Type $I$. A vector of the form $R(a, b, c)$ with $a+b \leq p-1$ and $c \neq 0$ is said to be of Type II.

Lemma 9 The span of all $p^{2}$ vectors of Type I has dimension $p^{2}-p+1$.

Proof. Since $p$ is odd, and since each row subblock meets exactly one column subblock of each column block, a vector of Type I is the all-one vector in exactly one column subblock of each column block, and is zero in all other column subblocks. Therefore, the rank of the matrix $A$ whose rows are all the $p^{2}$ vectors of Type I is the same as the rank of the matrix of order $p^{2}$ obtained from $A$ by suppressing all but one column in each column subblock. But by Definitions 1 and 2 , the latter matrix is precisely $H(2, p)^{T}$, whose rank is $p^{2}-p+1$ by Theorem 2 .

Let $B$ be the set of vectors consisting of all vectors of Type II and a basis of the span of the vectors of Type I.

Lemma $10 B$ consists of $\left(p^{3}+2 p^{2}-3 p+2\right) / 2$ vectors.

Proof. There are $(p+1) p(p-1) / 2$ vectors of Type II, and by Lemma 9 there are $p^{2}-p+1$ vectors in the basis of the span of the vectors of Type I, totaling $\left(p^{3}+2 p^{2}-3 p+2\right) / 2$ vectors. 
Lemma $11 B$ spans the row-space of $H(3, p)^{T}$.

Proof. Consider a row subblock $(a, b)$ satisfying $a+b \leq p-1$. Since all its rows $R(a, b, c)$ with $c \neq 0$ are of Type II, and since $\sum_{c} R(a, b, c)$ is of Type I and thus in $\operatorname{span} B$, it follows that all the rows of this subblock are in $\operatorname{span} B$. Therefore it is enough to prove that all rows $R(a, b, c)$ with $a+b \geq p$ are spanned by the rows $R(a, b, c)$ with $a+b \leq p-1$ and the vectors of Type I. We do this by induction on $a$. In fact, we prove that for each $a$, all the rows $R(a, b, c)$ with $a+b \geq p$ are spanned by the rows of the form $R\left(a^{\prime}, \cdot, \cdot\right)$ with $a^{\prime} \leq a-1$, the rows of the form $R\left(a, b^{\prime}, \cdot\right)$ with $a+b^{\prime} \leq p-1$, and the vectors of Type I of the form $R(a, b, 0)$ with $a+b \geq p$. We denote by $\mathcal{S}$ the span of the latter rows.

The basis of the induction for $a=1$ follows directly from Lemma 8. We assume now that $a \geq 2$, and apply Lemma 8 with $a^{\prime} \leq a-1$. Since the first summation in (6) is in $\mathcal{S}$, so is the second:

$$
\text { for } a^{\prime} \leq a-1, \quad \sum_{b^{\prime \prime}} R\left(a, b^{\prime \prime}, a^{\prime}\left(b^{\prime \prime}-b\right)+c\right) \in \mathcal{S} \text {. }
$$

In (7), let $a^{\prime}$ take two values in turn: $a^{\prime}=0$ and $a^{\prime}=a_{2}^{\prime}$ with $1 \leq a_{2}^{\prime} \leq a-1$, then add the two resulting equations to obtain:

$$
\text { for } 1 \leq a_{2}^{\prime} \leq a-1, \quad \sum_{b^{\prime \prime}} R\left(a, b^{\prime \prime}, c\right)+\sum_{b^{\prime \prime}} R\left(a, b^{\prime \prime}, a_{2}^{\prime}\left(b^{\prime \prime}-b\right)+c\right) \in \mathcal{S} .
$$

When $b^{\prime \prime}$ takes the value $b$ in both summations of (8), the two terms cancel out. Furthermore, the terms involving $b^{\prime \prime}$ such that $a+b^{\prime \prime} \leq p-1$ are in $\mathcal{S}$, and so:

$$
\text { for } 1 \leq a_{2}^{\prime} \leq a-1, \quad \sum_{\substack{p-a \leq b^{\prime \prime} \leq p-1 \\ b^{\prime \prime} \neq b}}\left[R\left(a, b^{\prime \prime}, c\right)+R\left(a, b^{\prime \prime}, a_{2}^{\prime}\left(b^{\prime \prime}-b\right)+c\right)\right] \in \mathcal{S}
$$

Now given $a$ and $a_{2}^{\prime} \in\{1, \ldots, a-1\}$, choose $b=p-a$. Then (9) represents $p$ linear equations (one equation for each value of $c$ ) in the $p(a-1)$ unknown rows $R\left(a, b^{\prime \prime}, \cdot\right)$ with $b^{\prime \prime} \in\{p-a+1, \ldots, p-1\}$. If we let $a_{2}^{\prime}$ take each value in $\{1, \ldots, a-1\}$, we obtain a total of $p(a-1)$ equations in our $p(a-1)$ unknown rows. It is not hard to check that the coefficient matrix of these equations is the matrix $M$ appearing in Lemma 6 .

For example, suppose $p=5$ and $a=3$. For $a_{2}^{\prime}=1$ and $c=0$, (9) reads

$$
R(3,3,0)+R(3,3,1)+R(3,4,0)+R(3,4,2) \in \mathcal{S}
$$

for $a_{2}^{\prime}=1$ and $c=1(9)$ reads

$$
R(3,3,1)+R(3,3,2)+R(3,4,1)+R(3,4,3) \in \mathcal{S}
$$

and so on. The coefficient matrix $M$ of all these equations is displayed below, where each column labelled by $\left(a, b^{\prime \prime}, c\right)$ (where $\left.b^{\prime \prime}=3,4, c=0,1,2,3,4\right)$ represents one of the $5(3-1)=10$ unknowns $R\left(a, b^{\prime \prime}, c\right)$, and each row labelled by $\left(a_{2}^{\prime}, c\right)$ (where $a_{2}^{\prime}=1,2$, 
$c=0,1,2,3,4)$ represents one of the $5(3-1)=10$ equations.

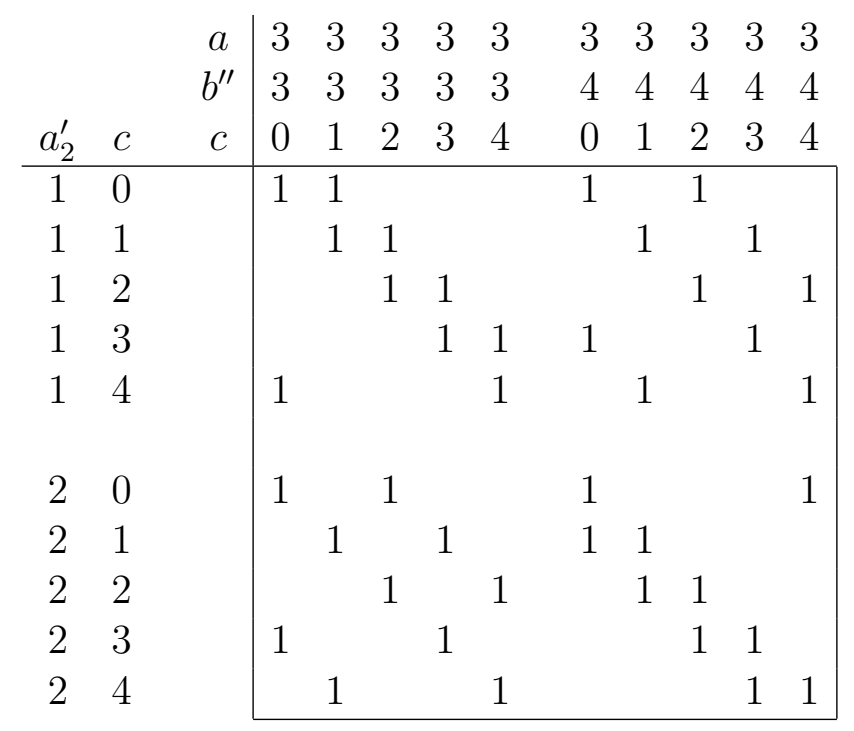

It is seen that in this example

$$
M=\left(\begin{array}{cc}
I_{0}+I_{1} & I_{0}+I_{2} \\
I_{0}+I_{2} & I_{0}+I_{4}
\end{array}\right),
$$

confirming the general form of the matrix $M$ in Lemma 6 .

By Lemma 6 , the rank of $M$ is $(p-1)(a-1)$ in general. Our unknown rows also satisfy the additional $a-1$ linear equations

$$
\text { for } b^{\prime \prime}=b+1, \ldots, b+a-1, \quad \sum_{c} R\left(a, b^{\prime \prime}, c\right) \in \mathcal{S}
$$

expressing the fact that the sum on the left is a vector of Type I. The coefficient matrix $N$ of the equations (10) has $a-1$ rows, each having the all-one vector in one block column and zero in all other block columns. In our above example where $q=5, a=3$

$$
N=\left(\begin{array}{rrrrrrrrrrr}
1 & 1 & 1 & 1 & 1 & & & & & \\
& & & & & & & & & & \\
& & & & & 1 & 1 & 1 & 1 & 1
\end{array}\right)
$$

Clearly the rows of $N$ are linearly independent, and furthermore, they are not spanned by the rows of $M$, since the rows of $N$ have odd weights and the rows of $M$ have even weights. So the combined coefficient matrix of the linear equations (9) and (10) in our $p(a-1)$ unknown rows has rank $(p-1)(a-1)+(a-1)=p(a-1)$. Therefore equations (9) and (10) express all the unknown rows of the form $R\left(a, b^{\prime \prime}, \cdot\right)$ with $b^{\prime \prime} \in\{p-a+1, \ldots, p-1\}$ as members of $\mathcal{S}$.

The remaining unknown rows for the given $a$ have the form $R(a, p-a, \cdot)$. To deal with them, we make another choice of $b$ in (9), namely $b=p-1$. When $b$ was $q-a$ and we let $b^{\prime \prime}$ run over its allowed values in $(9), b^{\prime \prime}-b$ ran over $1, \ldots, a-1$ in order; now that $b=p-1$, we see that $b^{\prime \prime}-b$ runs over $-a+1, \ldots,-1$ in order. In our example where $q=5, a=3$, the matrix of coefficients of the resulting equations is given by

$$
M^{\prime}=\left(\begin{array}{cc}
I_{0}+I_{-2} & I_{0}+I_{-1} \\
I_{0}+I_{-4} & I_{0}+I_{-2}
\end{array}\right),
$$


and in general $M^{\prime}$ has the same shape of $M$, but its $(i, j)$ block is $I_{0}+I_{-i(a-1-j)}$. It follows that $M^{\prime}$ is obtained from $M$ by reversing the order of the block columns, as well as the order of the columns in each block column and the order of the rows in each block row. Therefore $M$ and $M^{\prime}$ have the same rank, and the previous argument shows that all the unknown rows of the form $R\left(a, b^{\prime \prime}, \cdot\right)$ with $b^{\prime \prime} \in\{p-a, \ldots, p-2\}$ are also members of $\mathcal{S}$.

Proof of Theorem 6. The proof follows from Lemmas 10 and 11 and the fact that $H(3, p)^{T}$ has $p^{3}$ rows.

\section{Acknowledgments}

We thank Keith Mellinger for calling our attention to [13].

\section{References}

[1] E. F. Assmus Jr. and J. D. Key, Designs and Their Codes, Cambridge University Press, Cambridge, 1992.

[2] A. E. Brouwer, "Bounds on the size of linear codes," in Handbook of Coding Theory, V. S. Pless and W. C. Huffman, Editors, Elsevier, pp. 295-452, 1998.

[3] J. Cannon and C. Playoust, An Introduction to Magma, University of Sydney, Sydney, Australia, 1994.

[4] C. Di, D. Proietti, I. E. Telatar, T. J. Richardson and R. L. Urbanke, "Finite-Lenght Analysis of Low-Density Parity-Check codes on the Binary Erasure Channel," IEEE Trans. Infom. Theory, vol. 48, no. 6 pp. 1570-1579, June. 2002

[5] R. G. Gallager, "Low density parity check codes," IRE Trans. Infom. Theory, vol. IT8, pp.21-28, Jan. 1962

[6] J.-L. Kim, U. N. Peled, I. Perepelitsa and V. Pless, "Explicit construction of families of LDPC codes of girth at least six," Proc. 40th Allerton Conf. on Communication, Control and Computing, P. G. Voulgaris and R. Srikant, Eds., pp. 1024-1031, Oct. $2-4,2002$,

[7] Y. Kuo, S. Lin and M. P. C. Fossorier, "Low-density parity-check codes based on finite geometries: a rediscovery and new results," IEEE Trans. Inform. Theory, vol. 47, no. 7, pp. 2711-2736, Nov. 2001.

[8] E. S. Lander, Symmetric Designs: An Algebraic Approach, Cambridge University Press, Cambridge, 1983.

[9] F. Lazebnik and V. A. Ustimenko, "Explicit construction of graphs with arbitrary large girth and of large size," Discrete Applied Math., vol. 60, pp. 275-284, 1997.

[10] F. Lazebnik and A. J. Woldar, "General properties of some families of graphs defined by systems of equations," J. Graph Theory, vol. 38, pp. 65-86, 2001.

[11] D. J. C. MacKay and R. M. Neal, "Near Shannon limit performance of low density parity check codes," Electron. Lett., vol. 32, no. 18, pp. 1645-1646, 1996 
[12] R. M. Tanner, "A recursive approach to low complexity codes," IEEE Trans. Inform. Theory, vol. IT - 27, pp. 533-547, 1981.

[13] R. Viglione, "Properties of some algebraically defined graphs," Ph.D. Thesis, Univ. of Delaware, 2002. 